\title{
Johnson photometry of southern Blazhko targets
}

\author{
K. Kolenberg ${ }^{1,2}$, E. Guggenberger ${ }^{1}$, and T. Medupe ${ }^{3}$ \\ ${ }^{1}$ Institut für Astronomie, Türkenschanzstrasse 17, 1180 Vienna, Austria \\ 2 Instituut voor Sterrenkunde, Celestijnenlaan 200D, B-3001 Leuven, Belgium \\ ${ }^{3}$ University of Cape Town, South Africa
}

\begin{abstract}
We report on differential photomultiplier photometry in the Johnson $B$ and $V$ filters of five southern Blazhko stars (RU Cet, RY Col, V674 Cen, AR Ser, RV Cap). The data were gathered from the South African Astronomical Observatory (SAAO) in Sutherland, South Africa, in the course of 2004 and 2005. To find the most reliable values for the periods, we combined our $V$ data with ASAS photometry. For some stars, this enabled us to constrain their Blazhko period with unprecedented accuracy.

Individual Objects: RU Cet, RY Col, V674 Cen, AR Ser, RV Cap, SS For, UV Oct, HD 6022, SAO 147558, HD 33741, HD 33562, HD 123388, CD 359211, HD 138735, HD 138041, HD 200182, HD 200068
\end{abstract}

\section{Introduction}

Investigations of the pulsating RR Lyrae stars contribute to almost every branch of modern astronomy. These stars, with mean periods of about half a day and brightness variations of about one magnitude, have generally been considered to be prototypes of pure radial pulsation. They pulsate in the radial fundamental mode (type RRab), the radial first overtone (type $\mathrm{RRc}$ ), and both of these radial modes simultaneously (type RRd). However, additional cycles occur in many RR Lyrae stars. A considerable fraction of the RR Lyrae stars (20-30\% of Galactic RRab, 5\% of RRc stars) shows a periodic amplitude/phase modulation on timescales of typically tens to hundreds of times the pulsation period, denoted as the Blazhko effect (Blazhko 1907). More than a century after its discovery, the phenomenon remains a mystery.

The two most widely accepted and quoted hypotheses to explain the phenomenon invoke either a resonance effect (Nowakowski \& Dziembowski 2001; Van Hoolst et al. 1998; Dziembowski \& Mizerski 2004), or a magnetic field (Shibahashi \& Takata 1995). Both models involve nonradial pulsation components (Kovacs 2002). However, there are models around that do not require the presence of nonradial pulsation modes, such 
as the scenario proposed by Stothers (2006), in which a variable turbulent convection causes the observed amplitude modulation. But the truth is that till now none of the available models can satisfactorily explain the variety of observed characteristics of the Blazhko effect. The detection and identification of nonradial pulsation components, or the proof of their absence, remains crucial for understanding the mechanism behind the amplitude modulation.

The frequency spectra of light curves of RR Lyrae Blazhko stars exhibit either a doublet or an equally-spaced triplet structure, with a small frequency separation corresponding to the frequency of the modulation. Quintuplet structures, as predicted by the magnetic model, are rarely observed in Blazhko stars, though we should mention the exception of RV Uma (Hurta et al. 2008).

In 2004, we began a photometric survey of a well-selected sample of southern field Blazhko targets. Previously, most Blazhko studies had been carried out from the northern hemisphere (e.g., Szeidl 1988, and references therein; Smith 1995, and references therein; Borkowski 1980; Kovács 1995; Smith et al. 1994, just to mention a few). Therefore it was more difficult to find well-established field Blazhko stars at southern declinations. The available data of southern field Blazhko stars (e.g., Hoffmeister 1956; Clube et al. 1969; Lub 1977) are insufficient to determine the Blazhko periods with the required accuracy. Based on ASAS data, Wils \& Sodor (2005) published a list of new and confirmed Blazhko targets, and Szczygiel \& Fabrycky (2007) obtained interesting new results, including a star with multiple Blazhko periods. We also note that the Northern Sky Variability Survey (NSVS), obtained with the ROTSEI telescope (Woźniak et al. 2004), goes down to southern latitudes and contains valuable data for many RR Lyrae stars (Kinemuchi et al. 2006).

However, accurate and complete photometric data sets of southern field Blazhko stars, covering complete light curves at different Blazhko phases, were (and are in part) still lacking. Our photometric campaign was aimed at filling this void.

\section{Target selection}

Through inspection of several stellar data bases (ASAS ${ }^{1}$, GCVS $^{2}$, HIPPARCOS $^{3}$, AAVSO ${ }^{4}$, GEOS $^{5}$ ) and, if possible, additional photometric data (e.g., Clube et al. 1969; Lub 1977), we made a selection of the most interesting and rather bright ( $V \simeq 9$ to 11 mag) currently known field Blazhko stars, listed in Table 1. Note that the Blazhko periods $P_{\mathrm{Bl}}$ (old) listed in Table 1 and taken from the literature are often based on studies carried out decades ago, or on a limited amount of photometric data, often centered around maximum light. The Blazhko periods $P_{\mathrm{Bl}}$ (ASAS) listed in Table 1 are based on the analysis of the ASAS data (see also Wils \& Sodor 2005). These data have been obtained over a long time span (between 5.5 and 7.1 years for our targets) and are characterized by sporadic data points, typically every few nights, and

\footnotetext{
${ }^{1}$ http://archive.princeton.edu/ asas/

${ }^{2}$ http://www.sai.msu.su/groups/cluster/gcvs/gcvs/

${ }^{3} \mathrm{http}: / /$ www.rssd.esa.int/index.php?project=HIPPARCOS\&page=Epoch_Photometry

${ }^{4}$ http://www.aavso.org/vstar/

${ }^{5}$ http://dbrr.ast.obs-mip.fr/
} 
Table 1: List of our southern Blazhko targets with information on their pulsation properties. For consistency, all magnitude ranges and pulsation periods are taken from the General Catalogue of Variable Stars (Kholopov et al. 1998). The period determined from the ASAS data base may slightly differ from these.

\begin{tabular}{|c|c|c|c|c|c|}
\hline Name & $\begin{array}{c}V \\
(\mathrm{mag})\end{array}$ & $\begin{array}{l}P_{\text {puls }} \\
\text { (d) }\end{array}$ & $\begin{array}{c}P_{\mathrm{Bl}}(\mathrm{old}) \\
\text { (d) }\end{array}$ & $\begin{array}{c}P_{\mathrm{Bl}}(\mathrm{ASAS}) \\
(\mathrm{d})\end{array}$ & $\begin{array}{c}P_{\mathrm{Bl}}(\text { this study }) \\
\text { (d) }\end{array}$ \\
\hline RU Cet & $10.8-12.1$ & 0.58628 & - & 97 & $97.9 \pm 1.0$ \\
\hline SS For & $9.5-10.6$ & 0.495432 & - & $34.8^{a}$ & $34.7 \pm 0.2^{b}$ \\
\hline RY Col & $10.4-11.2$ & 0.4788571 & 90: & $82^{a}$ & $82.4 \pm 0.8$ \\
\hline V674 Cen & $11.0-11.9$ & 0.493919 & 29.5: & - & TBD \\
\hline AR Ser & $11.4-12.3$ & 0.5751416 & 105 & - & $110.0 \pm 5.0$ \\
\hline UV Oct & $8.7-9.9$ & 0.542625 & 82: & $145^{a}$ & $143.9 \pm 0.2^{b}$ \\
\hline RV Cap & $10.2-11.6$ & 0.44774401 & 225.5 & 233 & $232.6 \pm 5.5$ \\
\hline \multicolumn{6}{|c|}{$\begin{array}{l}": " \text { denotes uncertain period; } \\
a: \text { period also found by Wils \& Sodor }(2005) \text { in the ASAS data. } \\
b: \text { For these stars the detailed analysis will be published separately. } \\
\text { TBD: to be determined! }\end{array}$} \\
\hline
\end{tabular}

a rather large scatter. However, due to their long time span, the ASAS data can be helpful in determining an accurate mean period. The values $P_{\mathrm{Bl}}$ (this study) for the Blazhko period were determined by means of our dedicated photometric campaign in combination with ASAS data. Note that for RU Cet, SS For, V674 Cen, AR Ser, and RV Cap, there are also entries in the NSVS (Woźniak et al. 2004) but we did not include these data, which have not been obtained in the Johnson $V$ scale, in the analysis presented below. For two of our targets, SS For and UV Oct, we obtained highprecision photometry with a good spread over both the pulsation and Blazhko cycles, using telescopes at both SAAO (South Africa) and SSO (Siding Spring Observatory, Australia).

Large amounts of high-precision multisite data allowed to perform several additional analyses for those two stars that would go beyond the scope of this paper. Therefore, the outcome of these analyses will be published in separate articles. In this paper we focus on the other targets for which less extensive photometry was obtained (see Table 2). The data presented here are available online through the Blazhko website: http://www.univie.ac.at/tops/blazhko/data/.

\section{Observations and data reduction}

In 2004, 14 weeks (between Jan. 27 and Dec. 20, 2004) and, in 2005, 12 weeks (between Feb. 1 and Sept. 26, 2005) of observing time were allocated to this program at the South African Astronomical Observatory (SAAO) in Sutherland. The data were gathered using the three small telescopes on site: the $50-\mathrm{cm}$, the $75-\mathrm{cm}$, and the 1$\mathrm{m}$ telescope (1 week). Telescopes were allocated to the project depending on their 
Table 2: Observation log for our targets, listing the number of hours, the number of nights during which data were gathered, and the number of measurements, respectively in the $V$ and $B$ filter. Further, the total time span T (in days) of the observations is given for each of the targets for each year (2004 and 2005). Finally, the number of used ASAS data points and their time span $\mathrm{T}$ are given.

\begin{tabular}{lllllllll}
\hline \multicolumn{1}{c}{ Name } & Hours & Nights & Nr. $^{V}$ & Nr. $^{B}$ & $\mathrm{~T}^{04}$ & $\mathrm{~T}^{05}$ & Nr. $^{\text {ASAS }}$ & $\mathrm{T}^{\text {ASAS }}$ \\
\hline RU Cet & 17.87 & 4 & 123 & 122 & 11.1 & - & 297 & 2039.3 \\
RY Col & 57.95 & 24 & 293 & 301 & 70.0 & 13.1 & 419 & 2002.8 \\
V674 Cen & 77.11 & 30 & 469 & 441 & 93.9 & 10.1 & 609 & 2596.0 \\
AR Ser & 37.39 & 18 & 293 & 244 & 70.0 & 6.1 & 341 & 2430.6 \\
RV Cap & 55.87 & 21 & 372 & 254 & 6.0 & 24.0 & 462 & 2460.6 \\
\hline
\end{tabular}

availability. The observations of the targets discussed here were carried out by the authors: TM and his students, EG and KK. When we could not send observers from Vienna to South Africa, this program was also used to train the students from the University of North West in observation.

The data were obtained in the Johnson $B$ and $V$ filters. For about $50 \%$ of the observations the classical 3-star technique (Breger 1993) was applied; for the other half, the measurements of the comparison stars were unfortunately more sporadic, implying a lower quality of the data. The comparison stars we used for each of the stars are listed in Table 3. The data reduction was done by EG. When possible, the extinction coefficient was determined for each night individually using Bouguer plots. For the other nights standard values were used. The sky background brightness was monitored and subtracted from the data. Finally, differential photometry was achieved by subtracting interpolated values of the comparison stars from those of the variable star.

\section{Results for the targets}

\section{Description of the frequency analysis}

For each of the targets we performed a frequency analysis using Period04 (Lenz \& Breger 2005), a package applying single-frequency power spectra and simultaneous multifrequency sine-wave fitting. We determined the frequencies by means of a successive prewhitening strategy. After prewhitening with the main frequency and its harmonics, additional peaks usually emerged. Significant frequency peaks appearing in the vicinity of the main frequency or its harmonics are a typical feature for stars showing Blazhko modulation. For Blazhko stars, the a priori choice is made very often to describe the light curve variations by means of equidistant triplets at the main frequency and its harmonics, and we followed this strategy in our analysis. We applied multi-frequency 
fits to our data, according to the formula:

$$
\begin{aligned}
& f(t)=A_{0}+\sum_{k=1}^{n}\left[A_{k} \sin \left(2 \pi k f_{0} t+\phi_{k}\right)\right. \\
& +A_{k+} \sin \left(2 \pi\left(k f_{0}+f_{B}\right) t+\phi_{k+}\right) \\
& \left.+A_{k-} \sin \left(2 \pi\left(k f_{0}-f_{B}\right) t+\phi_{k-}\right)\right] \\
& +B_{0} \sin \left(2 \pi\left(f_{B} t+\phi_{B}\right)\right)
\end{aligned}
$$

where $f_{0}$ is the main pulsation frequency and $f_{B}$ is the Blazhko frequency. We calculated amplitudes and phases up to order $n$, for which the amplitudes of the higher-order harmonics are still above the significance level. Note that we also only calculated the fit including the significant side peak frequencies. For each of the stars, we list the relevant frequency components and their amplitudes in Tables 4 to 8; for those just below the significance level the amplitude values are listed in italics. As a significance criterion, we adopted a signal-to-noise ratio $(\mathrm{S} / \mathrm{N})$ of 4 for independent frequencies and 3.5 for combination frequencies (Breger et al. 1993). We calculated optimum values for the frequencies, their amplitudes and phases by minimizing the residuals of the fit given by Equation (1). Errors on these parameters were determined through extensive Monte Carlo simulations integrated in Period04 (Lenz \& Breger 2005). The obtained uncertainties on frequencies and amplitudes were confirmed by analyses of different subsets of the data.

Since $f_{B}$ does not always have a detectable amplitude, we fit the data with the linear combinations of two independent frequencies, $f_{0}$ and $f_{N}$, where $f_{N}$ is the side peak frequency near $f_{0}$ with the highest amplitude (Kolenberg et al. 2006). For the

Table 3: Variables, comparison stars and their coordinates.

\begin{tabular}{lllll}
\hline \multicolumn{1}{c}{ Name } & RA & Dec & attribute \\
\hline RU Cet & 010040 & -155724 & Var \\
HD 6022 & 010120 & -161740 & C1 \\
SAO 147558 & 005926.8 & -161412 & C2 \\
\hline RY Col & 051508 & -413742 & Var \\
HD 33741 & 051022 & -413524 & C1 \\
HD 33562 & 051701 & -421311 & C2 \\
\hline V674 Cen & 140324 & -362420 & Var \\
HD 123388 & 140807 & -365803 & C1 \\
CD 359211 & 140223 & -361759 & C2 \\
\hline AR Ser & 153058 & 025624 & Var \\
HD 138735 & 153350 & 022840 & C1 \\
HD 138041 & 152927 & 015037 & C2 \\
\hline RV Cap & 205842 & -152524 & Var \\
HD 200182 & 210227 & -152455 & C1 \\
HD 200068 & 210131 & -152129 & C2 \\
\hline
\end{tabular}


majority of the stars in our sample, $f_{N}$ corresponds to $f_{0}+f_{B}$, the higher-frequency side peak at $f_{0}$. All detected frequencies typical for the periodogram of a Blazhko star are the result of linear combinations of $f_{0}$ and $f_{N}$.

For none of the targets presented here we obtained data covering the Blazhko cycle. To obtain an optimal period determination, we combined our data in the $V$ filter with ASAS data of the star. (ASAS-3 data are recorded in the $V$ band.) For this purpose, both the $V$ data and the ASAS data were fitted with the same frequency set, containing the main frequency and its significant harmonics and side peak frequencies if detected. Subsequently, the ASAS data were shifted by the difference in zero points of the respective fits to the SAAO $V$ data. In the combined data set, the ASAS data were given a lower weight than our more accurate SAAO $V$ data. Finally, the frequencies were determined through successive prewhitening as described above.

Using the frequencies obtained through the combination of our SAAO $V$ data and the ASAS data, we subsequently analyzed the data in $B$ and $V$, with respect to the two comparison stars. This method excludes the introduction of false frequencies introduced by a variable comparison star, as those frequencies would show up in only one of the two data sets. If the Blazhko period was only partly covered with our SAAO data, fitting these with the side peak frequencies does not yield reliable amplitudes. When we did not find any meaningful side peaks, we simply performed a fit with the main frequency and its harmonics. This still allows us to compare the behaviour of the light variation in $B$ and $V$.

Quintuplet components have been detected recently and for the first time in the frequency spectrum of the Blazhko variable RV UMa (Hurta et al. 2008). We found no evidence of additional frequency peaks at the expected positions of the quintuplet components, but the noise level in our data typically lies at $0.005 \mathrm{mag}$. Note that Alcock et al. (2003) failed to clearly detect such components at the $\simeq 0.004 \mathrm{mag}$ level.

\section{RU Cet}

Early data of the star were published by Hoffmeister (1931), Robinson (1966), Clube et al. (1969), and Jones (1973). From the ASAS photometry, a Blazhko period of about 97 days emerges for RU Cet. This star is not included in the paper by Wils \& Sodor (2005). We only obtained data of RU Cet during 4 nights in 2004, including one maximum. This amount of data is clearly insufficient to fit the Blazhko period. After combining the $V$ data with ASAS photometry (279 data points over 2039.3 days), we obtain a main period of 0.586289 day and a Blazhko period of $97.9 \pm 1.0$ days.

The results of our analyses are listed in Table 4. Note that, due to insufficient data, it was impossible to fit the SAAO $V$ and $B$ data with the complete frequency set. We fitted the data in the Blazhko phase they were recorded. Thanks to the quality of the SAAO data, the harmonics of $f_{0}$ were significant up to the $10^{\text {th }}$ order. 

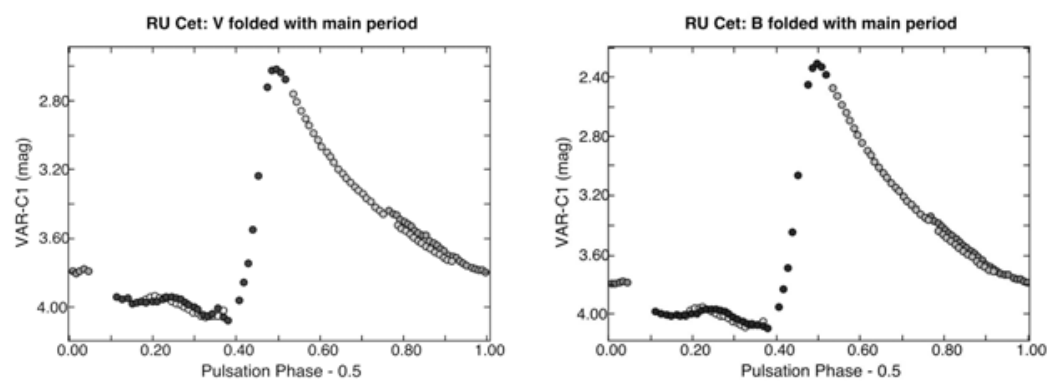

Figure 1: Our SAAO data on RU Cet.

Table 4: Results of our analysis with Period04 for RU Cet. The first column gives the relevant frequencies and their values, obtained with the combined data set. $A_{V+\text { ASAS }}$ gives the amplitudes for the combined data set, $A_{\mathrm{ASAS}}$ for ASAS only. $A_{V}$ and $A_{B}$ give the amplitudes obtained from our SAAO data. Note that we did not always manage to fit meaningfully with all the frequencies. For the SAAO data, we give the fit with the main frequency and its harmonics (mean light curve). For frequency peaks below the significance level, the values are listed in italics.

\begin{tabular}{|c|c|c|c|c|c|}
\hline & $\begin{array}{l}f \\
(\mathrm{c} / \mathrm{d})\end{array}$ & $\begin{array}{l}A_{V+\mathrm{ASAS}} \\
(\mathrm{mag})\end{array}$ & $\begin{array}{l}A_{\mathrm{ASAS}} \\
(\mathrm{mag})\end{array}$ & $\begin{array}{l}A_{V} \\
(\mathrm{mag})\end{array}$ & $\begin{array}{l}A_{B} \\
(\mathrm{mag})\end{array}$ \\
\hline$f_{0}$ & $1.70565^{(a)}$ & $0.38^{(c)}$ & 0.38 & $0.465^{(d)}$ & $0.599^{(e)}$ \\
\hline $2 f_{0}$ & 3.41131 & 0.17 & 0.17 & 0.258 & 0.311 \\
\hline $3 f_{0}$ & 5.11695 & 0.09 & 0.10 & 0.161 & 0.197 \\
\hline $4 f_{0}$ & 6.82260 & 0.06 & 0.07 & 0.131 & 0.162 \\
\hline $5 f_{0}$ & 8.52821 & 0.04 & 0.03 & 0.082 & 0.093 \\
\hline $6 f_{0}$ & 10.23390 & 0.02 & 0.02 & 0.048 & 0.055 \\
\hline $7 f_{0}$ & 11.93956 & & & 0.051 & 0.043 \\
\hline $8 f_{0}$ & 13.64525 & & & 0.029 & 0.027 \\
\hline $9 f_{0}$ & 15.35085 & & & 0.021 & 0.019 \\
\hline $10 f_{0}$ & 17.05650 & & & 0.018 & 0.011 \\
\hline$f_{0}+f_{B}$ & $1.71586^{(b)}$ & 0.05 & 0.10 & & \\
\hline$f_{0}-f_{B}$ & 1.69542 & 0.04 & 0.10 & & \\
\hline $2 f_{0}+f_{B}$ & 3.42151 & 0.04 & 0.13 & & \\
\hline $2 f_{0}-f_{B}$ & 3.40109 & 0.03 & 0.08 & & \\
\hline $3 f_{0}+f_{B}$ & 5.12716 & 0.04 & 0.07 & & \\
\hline $3 f_{0}-f_{B}$ & 5.10674 & 0.03 & 0.05 & & \\
\hline $4 f_{0}+f_{B}$ & 6.83281 & 0.03 & 0.07 & & \\
\hline $4 f_{0}-f_{B}$ & 6.81239 & 0.03 & 0.05 & & \\
\hline$f_{B}$ & 0.01021 & 0.005 & 0.005 & & \\
\hline \multicolumn{6}{|c|}{$\begin{array}{l}\text { Errors from MC: }{ }^{(a)}: \pm 0.00001 \mathrm{c} / \mathrm{d} ;{ }^{(b)}: \pm 0.0001 \mathrm{c} / \mathrm{d} ;{ }^{(c)}: \pm 0.01 \mathrm{mag} \\
\text { Errors from MC: }{ }^{(d)}: \pm 0.006 \mathrm{mag} ;(e): \pm 0.003 \mathrm{mag} \\
\text { Resulting Blazhko period: } 97.9 \pm 1.0 \text { days. }\end{array}$} \\
\hline
\end{tabular}



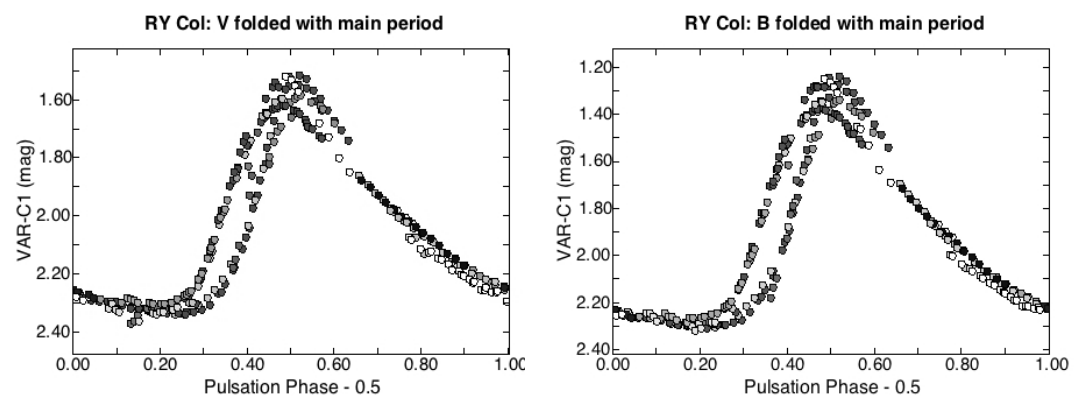

Figure 2: Our SAAO data on RY Col.

Table 5: Results of our analysis with Period04 for RY Col. The first column gives the relevant frequencies and their values, obtained with the combined data set. $A_{V+\mathrm{ASAS}}$ gives the amplitudes for the combined data set, $A_{\mathrm{ASAS}}$ for ASAS only. $A_{V}$ and $A_{B}$ give the amplitudes obtained from our SAAO data. For frequency peaks below the significance level the values are listed in italics.

\begin{tabular}{ll|ll|ll}
\hline$f$ & $\begin{array}{l}A_{V+\mathrm{ASAS}} \\
(\mathrm{mag})\end{array}$ & $\begin{array}{l}A_{\mathrm{ASAS}} \\
(\mathrm{mag})\end{array}$ & $\begin{array}{l}A_{V} \\
(\mathrm{mag})\end{array}$ & $\begin{array}{l}A_{B} \\
(\mathrm{mag})\end{array}$ \\
\hline$f_{0}$ & $2.08839^{(a)}$ & $0.36^{(c)}$ & 0.36 & $0.34^{(d)}$ & $0.40^{(e)}$ \\
$2 f_{0}$ & 4.17678 & 0.15 & 0.14 & 0.13 & 0.18 \\
$3 f_{0}$ & 6.26517 & 0.08 & 0.08 & 0.10 & 0.13 \\
$4 f_{0}$ & 8.35357 & 0.03 & 0.04 & 0.03 & 0.06 \\
$5 f_{0}$ & 10.44196 & 0.01 & 0.02 & 0.005 & 0.005 \\
$6 f_{0}$ & 12.53035 & 0.01 & 0.01 & 0.002 & 0.004 \\
$f_{0}+f_{B}$ & $2.10053^{(b)}$ & 0.07 & 0.07 & 0.07 & 0.04 \\
$f_{0}-f_{B}$ & 2.07625 & 0.03 & 0.03 & 0.06 & 0.07 \\
$2 f_{0}+f_{B}$ & 4.18894 & 0.06 & 0.06 & 0.04 & 0.07 \\
$2 f_{0}-f_{B}$ & 4.16462 & 0.03 & 0.03 & 0.03 & 0.06 \\
$3 f_{0}+f_{B}$ & 6.27734 & 0.05 & 0.05 & 0.06 & 0.06 \\
$3 f_{0}-f_{B}$ & 6.25302 & 0.02 & 0.03 & 0.02 & 0.03 \\
$4 f_{0}+f_{B}$ & 8.36571 & 0.03 & 0.03 & 0.03 & 0.05 \\
$4 f_{0}-f_{B}$ & 8.34140 & 0.02 & 0.02 & 0.01 & 0.05 \\
$f_{B}$ & 0.01214 & 0.01 & 0.002 & 0.02 & 0.03 \\
\hline
\end{tabular}

Errors from MC: ${ }^{(a)}: \pm 0.000005 \mathrm{c} / \mathrm{d} ;{ }^{(b)}: \pm 0.00006 \mathrm{c} / \mathrm{d} ;{ }^{(c)}: \pm 0.01 \mathrm{mag}$ Errors from MC: ${ }^{(d)}: \pm 0.02 \mathrm{mag} ;{ }^{(e)}: \pm 0.02 \mathrm{mag}$

Resulting Blazhko period: $82.4 \pm 0.8$ days. 

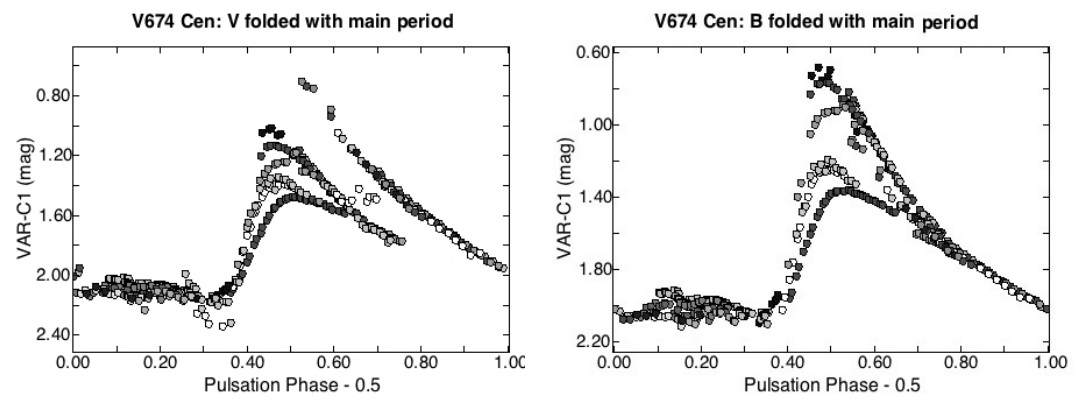

Figure 3: Our SAAO data on V674Cen.

Table 6: Results of our analysis with Period04 for V674 Cen. The first column gives the relevant frequencies and their values, obtained with the combined data set. $A_{V+\text { ASAS }}$ gives the amplitudes for the combined data set, $A_{\mathrm{ASAS}}$ for ASAS only. $A_{V}$ and $A_{B}$ give the amplitudes obtained from our SAAO data. For frequency peaks below the significance level the values are listed in italics. Errors based on Monte Carlo simulations are given below the table.

\begin{tabular}{ll|ll|ll}
\hline & $\begin{array}{l}f \\
(\mathrm{c} / \mathrm{d})\end{array}$ & $\begin{array}{l}A_{V+\mathrm{ASAS}} \\
(\mathrm{mag})\end{array}$ & $\begin{array}{l}A_{\mathrm{ASAS}} \\
(\mathrm{mag})\end{array}$ & $\begin{array}{l}A_{V} \\
(\mathrm{mag})\end{array}$ & $\begin{array}{l}A_{B} \\
(\mathrm{mag})\end{array}$ \\
\hline$f_{0}$ & $2.02438^{(a)}$ & $0.37^{(c)}$ & 0.37 & $0.39^{(d)}$ & $0.43^{(e)}$ \\
$2 f_{0}$ & 4.04877 & 0.12 & 0.14 & 0.11 & 0.18 \\
$3 f_{0}$ & 6.07313 & 0.09 & 0.10 & 0.12 & 0.12 \\
$4 f_{0}$ & 8.09751 & 0.06 & 0.05 & 0.05 & 0.04 \\
$5 f_{0}$ & 10.12189 & 0.04 & 0.04 & 0.04 & 0.04 \\
\hline
\end{tabular}




\section{RY Col}

First reported observations of this star were by Hoffmeister (1956) and it was classified as an RR Lyrae variable by Eggen (1956). Kinman (1961) published observations of the star, as well as Sturch (1966) and Lub (1977) who reported on the strong Blazhko effect of the star. In the ASAS data (419 points over 2002.8 days), we found a Blazhko period of $82.0 \pm 1.0$ days, in agreement with the value found by Wils \& Sódor (2005).

The results of our period analysis are given in Table 5 and a folded light curve of our $V$ and $B$ data is shown in Figure 2. We find a period of 0.478837 day and a Blazhko period of $82.4 \pm 0.8$ days.

\section{V674 Cen}

There are not many publications on the pulsation behavior of this star. It is first mentioned in Hoffmeister's (1956) paper on the light variations of short-periodic southern stars. Epstein \& Epstein (1973) publish 4-color photometric data, but insufficient to cover the light variation. Szeidl (1988) lists the star as having a Blazhko period of 29.5 days, but marks the period as uncertain.

We analyzed the ASAS data available for this star (604 points over 2596.0 days) and found no indication for a Blazhko period. From our SAAO data, however, it is clear that the star shows a strong Blazhko effect (Figure 3). Frequency peaks are found close to the main frequency and/or its harmonics, but none of them seems to point at a realistic Blazhko period (e.g., a side peak frequency of $4.04945 \mathrm{c} / \mathrm{d}$ next to $2 f_{0}$ would correspond to a period of 1448.5 days). As we find no reliable side peak frequencies, we present the fit to the data with the main frequency and its harmonics (Table 6 ). We find a main period of 0.493972 day.

\section{AR Ser}

AR Ser was discovered as a variable by Hoffmeister (1931). Szeidl (1988) reports on a secondary period of approximately 105 days. The star was also observed by Tsessevich (1977). Firmanyuk (1977) reports a main pulsation period of 0.575092 days $(1.73885 \mathrm{c} / \mathrm{d})$ and a Blazhko period of 108.4 days. The ASAS data on AR Ser (341 data points over 2430.6 days) yield no side peaks that are clearly reminiscent of a Blazhko effect, hence the star was not included in the list by Wils \& Sódor (2005). Our SAAO data, however, show a strong Blazhko effect (Figure 4). In the SAAO $V$ data, after subtracting the main frequency $f_{0}=1.73852 \mathrm{c} / \mathrm{d}$ and its harmonics, a peak emerges at $3.7471 \mathrm{c} / \mathrm{d}$, which could be interpreted as a 1-day alias of 1.7471 $\mathrm{c} / \mathrm{d}$. It is, however, not convincing.

Also in the combined data set, side peaks related to the Blazhko frequencies are hard to spot in the frequency spectrum after prewhitening with the main frequency and its harmonics. Two peaks appear at $1.73926 \mathrm{c} / \mathrm{d}$ and at $3.47776 \mathrm{c} / \mathrm{d}$, very close to $f_{0}$ and $2 f_{0}$, both corresponding to a period of 3.8-3.9 years. It remains to be proven whether these peaks are significant or an artefact caused by the sampling of our data set. After subtracting these very close side peaks, however, a frequency emerges at $1.74761 \mathrm{c} / \mathrm{d}$, at $0.00909 \mathrm{c} / \mathrm{d}$ distance from $f_{0}=1.73852 \mathrm{c} / \mathrm{d}$. This side peak corresponds with a Blazhko period of about 110 days. We find a period of 0.575202 day and a Blazhko period of $110.0 \pm 5.5$ days. 

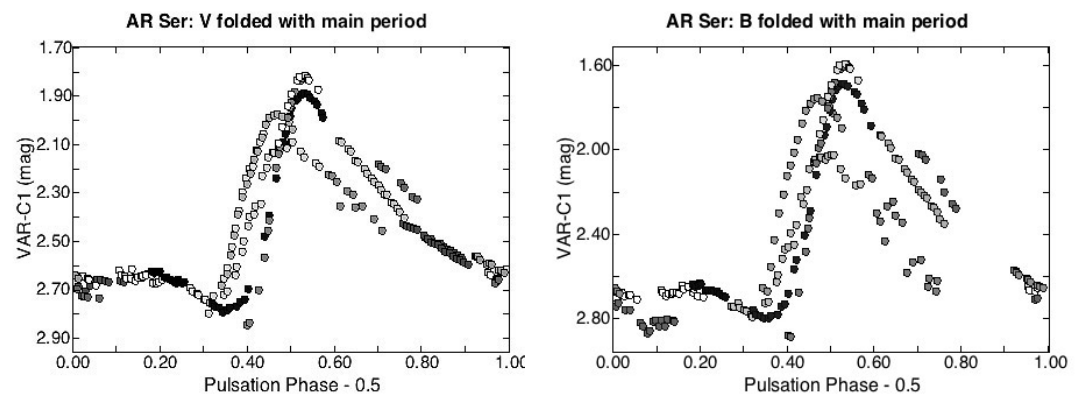

Figure 4: Our SAAO data on AR Ser.

Table 7: Results of our analysis with Period04 for AR Ser. The first column gives the relevant frequencies and their values, obtained with the combined data set. $A_{V+\text { ASAS }}$ gives the amplitudes for the combined data set, $A_{\mathrm{ASAS}}$ for ASAS only. $A_{V}$ and $A_{B}$ give the amplitudes obtained from our SAAO data. Note that we did not always manage to fit meaningfully with all the frequencies. For the SAAO data we give the fit with the main frequency and its harmonics (mean light curve). For frequency peaks below the significance level the values are listed in italics. Errors based on Monte Carlo simulations are given below the table.

\begin{tabular}{ll|ll|ll}
\hline & $\begin{array}{l}f \\
(\mathrm{c} / \mathrm{d})\end{array}$ & $\begin{array}{l}A_{V+\mathrm{ASAS}} \\
(\mathrm{mag})\end{array}$ & $\begin{array}{l}A_{\mathrm{ASAS}} \\
(\mathrm{mag})\end{array}$ & $\begin{array}{l}A_{V} \\
(\mathrm{mag})\end{array}$ & $\begin{array}{l}A_{B} \\
(\mathrm{mag})\end{array}$ \\
\hline$f_{0}$ & $1.73852^{(a)}$ & $0.26^{(c)}$ & 0.26 & $0.34{ }^{(d)}$ & $0.50^{(e)}$ \\
$2 f_{0}$ & 3.47705 & 0.09 & 0.08 & 0.12 & 0.17 \\
$3 f_{0}$ & 5.21626 & 0.06 & 0.06 & 0.13 & 0.14 \\
$4 f_{0}$ & 6.95410 & 0.01 & 0.03 & 0.03 & 0.08 \\
$5 f_{0}$ & 8.69258 & 0.02 & 0.02 & 0.03 & 0.01 \\
$f_{0}+f_{B}$ & $1.7476^{(b)}$ & 0.06 & 0.06 & & \\
$f_{0}-f_{B}$ & 1.7297 & 0.04 & 0.04 & & \\
$2 f_{0}+f_{B}$ & 3.4859 & 0.03 & 0.03 & \\
$2 f_{0}-f_{B}$ & 3.4681 & 0.02 & 0.02 & \\
\hline & Errors from MC: ${ }^{(a)}: \pm 0.00001 \mathrm{c} / \mathrm{d} ;{ }^{(b)}: \pm 0.0001 \mathrm{c} / \mathrm{d} ;{ }^{(c)}: \pm 0.01 \mathrm{mag}$ \\
Errors from MC: ${ }^{(d)}: \pm 0.01 \mathrm{mag} ;{ }^{(e)}: \pm 0.01 \mathrm{mag}$ \\
\multicolumn{5}{l}{ Resulting Blazhko period: $110.0 \pm 5.0 \mathrm{days}}$.
\end{tabular}

We refrain from presenting the fit to the data with additional side peak frequencies, as we feel more data are needed for more reliable results. For the same reason, we only fit the SAAO $V$ and $B$ data with a mean light curve containing the main frequency and its harmonics (Table 7 ). 


\section{RV Cap}

The reports on the actual Blazhko period of the star have been varied. Based on observations carried out in Catania between 1970 and 1972, Kanyo (1972) found a secondary period for the star of 138.3 days, which fits better than what Tsessevich (1953) found: 221.86 days. Szeidl (1988) reports a Blazhko period of 225.2 days.

At first sight, the new SAAO data do not look encouraging for a determination of the Blazhko period, as we obtained data in 6 nights in 2004 around what seems minimum Blazhko amplitude, and in 15 nights in 2005 we observed the star while its light amplitude went down. In the ASAS data (462 data points over 2460.6 days) we find, after prewhitening with the main frequency and its harmonics, two side peak frequencies at $f_{0}+f_{B}(2.2377 \mathrm{c} / \mathrm{d})$ and at $2 f_{0}+f_{B}(4.4711 \mathrm{c} / \mathrm{d})$. These both correspond to a Blazhko period of about 232.6 days.

After prewhitening with the main frequency and its harmonics in the combined data set, we find clear sidepeaks in the data (Figure 5). First, we find the right side peaks at $f_{0}+f_{B}(2.2377 \mathrm{c} / \mathrm{d}), 3 f_{0}+f_{B}(6.7046 \mathrm{c} / \mathrm{d})$, and $2 f_{0}+f_{B}(4.4711 \mathrm{c} / \mathrm{d})$, through successive prewhitening. Subsequently, we find the left side peaks at $f_{0}-f_{B}$ $(2.2291 \mathrm{c} / \mathrm{d}), 2 f_{0}-f_{B}(4.4626 \mathrm{c} / \mathrm{d})$, then $4 f_{0}+f_{B}(8.9380 \mathrm{c} / \mathrm{d})$, etc. All side peaks are found with frequency values within the error bars of the position of the expected triplet components. We find a main period of 0.447743 day and a Blazhko period of $232.6 \pm 5.5$ days. The results of our period analysis are given in Table 8 and a folded light curve of our $V$ and $B$ data is shown in Figure 6 . Note that the star long considered to have the longest known Blazhko period, turns out to have an even longer Blazhko period!

Note also the Blazhko frequency itself $\left(f_{B}=0.0043 \mathrm{c} / \mathrm{d}\right)$ has a small but significant amplitude in the Fourier spectrum. Additional frequencies can be found above the noise level in the combined data set. We could not prove nor disprove their reality on the basis of our data.
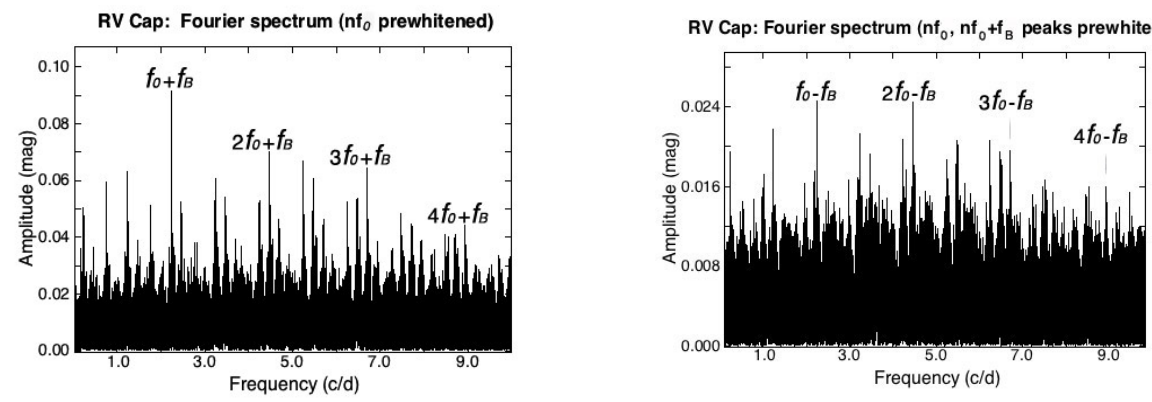

Figure 5: Fourier spectrum of the combined SAAO $V$ and ASAS data. Left panel: after prewhitening with the main frequency and its harmonics the right side peaks clearly appear in the spectrum. Right panel: after prewhitening with the main frequency, its harmonics and the right side peaks, the left side peaks appear in the spectrum. 

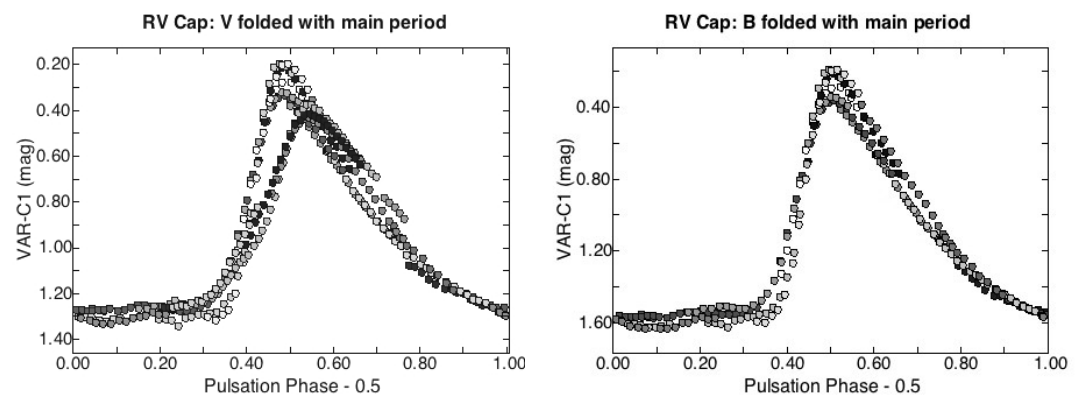

Figure 6: Our SAAO data on RV Cap. We obtained more data in $V$ than in $B$.

Table 8: Results of our analysis with Period04 for RV Cap. The first column gives the relevant frequencies and their values, obtained with the combined data set. $A_{V+\text { ASAS }}$ gives the amplitudes for the combined data set, $A_{\mathrm{ASAS}}$ for ASAS only. $A_{V}$ and $A_{B}$ give the amplitudes obtained from our SAAO data. Note that we did not always manage to fit meaningfully with all the frequencies. The fit to the $V$ and $B$ data in the last two columns was performed to the 2005 data only; we give the fit with the main frequency and its harmonics (mean light curve). For frequency peaks below the significance level the values are listed in italics.

\begin{tabular}{|c|c|c|c|c|c|}
\hline & $\begin{array}{l}f \\
(\mathrm{c} / \mathrm{d})\end{array}$ & $\begin{array}{l}A_{V+\mathrm{ASAS}} \\
(\mathrm{mag})\end{array}$ & $\begin{array}{l}A_{\mathrm{ASAS}} \\
(\mathrm{mag})\end{array}$ & $\begin{array}{l}A_{V} \\
(\mathrm{mag})\end{array}$ & $\begin{array}{l}A_{B} \\
(\mathrm{mag})\end{array}$ \\
\hline$f_{0}$ & $2.23342^{(a)}$ & $0.41^{(c)}$ & 0.40 & $0.424^{(d)}$ & $0.547^{(e)}$ \\
\hline $2 f_{0}$ & 4.46684 & 0.16 & 0.15 & 0.183 & 0.230 \\
\hline $3 f_{0}$ & 6.70026 & 0.07 & 0.06 & 0.106 & 0.133 \\
\hline $4 f_{0}$ & 8.93366 & 0.04 & 0.03 & 0.063 & 0.079 \\
\hline $5 f_{0}$ & 11.16720 & 0.02 & 0.02 & 0.035 & 0.045 \\
\hline $6 f_{0}$ & 13.40061 & 0.01 & 0.01 & 0.023 & 0.030 \\
\hline$f_{0}+f_{B}$ & $2.2377^{(b)}$ & 0.11 & 0.10 & & \\
\hline$f_{0}-f_{B}$ & 2.2291 & 0.06 & 0.06 & & \\
\hline $2 f_{0}+f_{B}$ & 4.4711 & 0.08 & 0.07 & & \\
\hline $2 f_{0}-f_{B}$ & 4.4626 & 0.05 & 0.06 & & \\
\hline $3 f_{0}+f_{B}$ & 6.7046 & 0.07 & 0.06 & & \\
\hline $3 f_{0}-f_{B}$ & 6.6960 & 0.03 & 0.04 & & \\
\hline $4 f_{0}+f_{B}$ & 8.9380 & 0.05 & 0.04 & & \\
\hline $4 f_{0}-f_{B}$ & 8.9294 & 0.01 & 0.02 & & \\
\hline $5 f_{0}+f_{B}$ & 11.1714 & 0.03 & 0.02 & & \\
\hline $5 f_{0}-f_{B}$ & 11.1628 & 0.01 & 0.02 & & \\
\hline $6 f_{0}+f_{B}$ & 13.4049 & 0.02 & 0.01 & & \\
\hline $6 f_{0}-f_{B}$ & 13.3963 & 0.01 & 0.01 & & \\
\hline$f_{B}$ & 0.0043 & 0.02 & 0.02 & & \\
\hline \multicolumn{6}{|c|}{$\begin{array}{l}\text { Errors from MC: }{ }^{(a)}: \pm 0.00001 \mathrm{c} / \mathrm{d} ;{ }^{(b)}: \pm 0.0001 \mathrm{c} / \mathrm{d} ;{ }^{(c)}: \pm 0.01 \mathrm{mag} \\
\text { Errors from MC: }{ }^{(d)}: \pm 0.006 \mathrm{mag} ;{ }^{(e)}: \pm 0.006 \mathrm{mag} \\
\text { Resulting Blazhko period: } 232.6 \pm 5.5 \text { days. }\end{array}$} \\
\hline
\end{tabular}


As we only obtained $B$ data in 2005, we fitted both $B$ and $V$ in 2005 with the main frequency and its harmonics to allow a comparison of their amplitudes. In 2005, we observed only part of the descending light phases of RV Cap, hence fitting with the side peak frequencies makes no sense, as this would lead to spurious amplitude values.

\section{Discussion}

Some thoughts arising from our analysis are summarized below.

- Thanks to the combination of the long time span of the ASAS data, and the better light curve coverage of our SAAO data, the frequencies could be obtained more accurately. We were able to find more accurate Blazhko periods from the combined data set, whereas we were not able to find them in the separate samples. For 4 of the 5 targets presented here, we were able to refine the Blazhko period with unprecedented accuracy. In the determination of the Blazhko period, we started from the assumption that the stars' variations can be modeled with equidistant triplet components around the main frequency and its harmonics. Note that it is possible that the Blazhko period has changed slightly in the course of our observations, though our data set does not allow to verify this. For several stars (XZ Cyg - LaCluyzé et al. 2004; RR Lyr Kolenberg et al. 2006) changing Blazhko periods have been observed.

- It is a statistical fact that the right side peaks in the multiplets are more often higher. In the 731 MACHO stars examined by Alcock et al. (2003), this occurred in about $75 \%$ of the studied cases. In our small sample of stars, this also turns out to be the case.

- With our data we can also confirm the finding by Jurcsik et al. (2005a) that the harmonics of the main frequency $f_{0}$ have a much steeper decrease in amplitude than the side peaks frequencies detected around the harmonics. In some cases the side peaks at $4 f_{0}$ and $5 f_{0}$ are still significant whereas the harmonics themselves are hidden in the noise.

- A search for a modulation component with the Blazhko period was successful in some cases (e.g., RV Cap). The MACHO Blazhko variables (Alcock et al. 2003) showed such a modulation with an overall amplitude of $\simeq 0.006 \mathrm{mag}$.

- Assuming that our SAAO $B$ and $V$ data were obtained with a good spread over the pulsation cycle and at similar phases, we can compare their amplitudes. In the components representing the main frequency and its harmonics, we find a ratio $A_{B} / A_{V}$ of typically $1.20-1.45$. Accordingly, Blazhko modulation in the $B$ filter is larger than in the $V$ filter (see also Jurcsik et al. 2005b).

- As we covered only part of a Blazhko cycle with our data, the presented stars are worth a follow-up study. Especially V674 Cen, for which we could clearly demonstrate the Blazhko effect but not determine its period, a (short) dedicated campaign will yield new results. 
Table 9: Ephemerides for our five targets on the basis of our analysis.

\begin{tabular}{lccl}
\multicolumn{1}{c}{ Name } & $\begin{array}{c}\text { HJD }\left(T_{\max }\right) \\
-2450000\end{array}$ & $\begin{array}{c}P_{0} \\
(\mathrm{~d})\end{array}$ & \multicolumn{1}{c}{$\begin{array}{c}P_{B} \\
(\mathrm{~d})\end{array}$} \\
\hline RU Cet & $3291.581 \pm 0.002$ & 0.586289 & $97.9 \pm 1.0$ \\
RY Col & $3415.426 \pm 0.002$ & 0.478837 & $82.4 \pm 0.8$ \\
V674 Cen & $3087.354 \pm 0.004$ & 0.493972 & $?$ \\
AR Ser & $3059.604 \pm 0.002$ & 0.575202 & $110.0 \pm 2.0$ \\
RV Cap & $3575.488 \pm 0.002$ & 0.447743 & $232.6 \pm 5.5$ \\
\hline
\end{tabular}

- On the basis of our data we can provide ephemerides for the observed targets (Table 9). We list the time of maximum light $\operatorname{HJD}\left(T_{\max }\right)$, the pulsation period and the Blazhko period (see also Table 1 ). The times of maximum Blazhko amplitude are not listed in Table 9.

\section{Continuation}

This observing run was carried out in the framework of an international collaboration focused on understanding the Blazhko effect (the 'Blazhko project ${ }^{6}$ ).

From this photometric campaign, we selected the best targets for a detailed spectroscopic study. It is clear that a photometric follow-up campaign on southern Blazhko targets would be useful. In this regard, it is regrettable that small professional telescopes, such as the ones used for this study, are gradually closing down in the larger professional observatories on both hemispheres. These telescopes are very well suited for variable star research and for training astronomy students. However, from different angles the study of southern Blazhko stars continues. Thanks to small robotic telescopes such as TAROT ${ }^{7}$ the times of maximum light of Blazhko stars are monitored from both hemispheres (LeBorgne, Klotz \& Boer 2007). Time has already been granted at various robotic facilities (TAROT, REM ${ }^{8}$ ) in Chile to continue the survey of southern Blazhko stars in 2008.

Acknowledgments. This paper uses observations made at the South African Astronomical Observatory (SAAO). We consulted several data bases in the course of this project: ASAS, SIMBAD, GEOS, GCVS, HIPPARCOS). KK and EG are supported by the Austrian Research Fund, FWF, projects T359 and P19962. KK cordially thanks Jan Lub for providing us with his older data in preparation of this project, Geza Kovács for his help and suggestions for target selection on the basis of ASAS, Bela Szeidl for sharing his knowledge on particular stars, and the staff at SAAO, especially Di Cooper and Chris Koen, for their flexibility in allocating time for this project. We thank Michel Breger for fruitful discussions. We thank the referee for helpful suggestions.

\footnotetext{
${ }^{6}$ http://www.univie.ac.at/tops/blazhko/

${ }^{7}$ http://tarot.obs-hp.fr/

${ }^{8}$ http://www.rem.inaf.it/
} 


\section{References}

Alcock, C., Alves, D. R., Becker, A., et al. 2003, ApJ 598, 597

Blazhko, S. 1907, AN 175, 325

Borkowski, K. J. 1980, AcA 30, 393

Breger, M. 1993, Proc. IAU Coll. No. 136, 106

Breger, M., Stich, J., Garrido, R., et al. 1993, A\&A 271, 482

Clube, S. V. M., Evans, D.S., Jones, D. H. P. 1969, MNRAS 72, 101

Dziembowski, W.A., \& Mizerski, T. 2004, AcA 54, 363

Eggen, O. J., 1956, PASP 68, 142

Epstein, I., \& Epstein, A. E. A. 1973, AJ 78, 83

Firmanyuk, B. N. 1977, IBVS 1245, 1

Hoffmeister, C. 1931, AN 242, 129

Hoffmeister, C. 1956, VeSon 3, 1

Hurta, Zs., Jurcsik, J., Szeidl, B. \& Sodor, A. 2008, arXiv:astro-ph/0712.0479v1 Jones, D. H.P. 1973, ApJSS 25, 487

Jurcsik J., Sódor, Á., Váradi, M., et al. 2005a, A\&A 430, 1049

Jurcsik J., Szeidl B., Nagy A., \& Sodor A. 2005b, IBVS 5666

Kanyo, S. 1972, IBVS 737, 1

Kinman, T. D. 1961, R. Obs. Bull. 37, 151

Kholopov, P. N.,Samus, N. N., Frolov, M.S., et al. 1998, Combined General Catalogue of Variable Stars, 4.1 Ed (II/214A)

Kinemuchi, K., Smith, H. A., Woźniak, P. R., \& McKay, T. A. 2006, AJ 132, 1202

Kolenberg, K., Smith, H. A., Gazeas, K. D., et al. 2006, A\&A 459, 577

Kovács, G. 1995, A\&A 295, 693

Kovács, G. 2002, ASPC 259, 396

LeBorgne, J. F., Klotz, A., \& Boer, M. 2007, IBVS 5790

LaCluyz, A., Smith, H. A., Gill, E.-M., et al. 2004, AJ 127, 1653

Lenz, P., \& Breger, M. 2005, CoAst 146, 53

Lub, J. 1977, A\&A 29, 345

Nowakowski, R. M., \& Dziembowski, W. A. 2001, AcA 51, 5

Robinson L. 1966, IBVS, 1

Shibahashi, H., \& Takata, M. 1995, ASPC 83, 42

Smith, H. A. 1995, "RR Lyrae Stars", Cambridge Univ. Press

Smith, H. A., Matthews, J. M., Lee, K. M., et al. 1994, AJ 107, 679

Stothers, R. B. 2006, ApJ 652, 643

Sturch, C. 1966, ApJ 143, 774 
Szczygiel, D. M., \& Fabrycky, D. C. 2007, MNRAS 377, 1263

Szeidl, B. 1988, in Multimode Stellar Pulsations, 45

Tsessevich, V.P. 1953, Trudi GAIS 23

Tsessevich, V.P. 1977, Perem. Zvezdy Prilozh., 3, 93

Van Hoolst, T., Dziembowski, W. A., Kawaler, S. D. 1998, MNRAS 297, 536

Woźniak, P. R., Vestrand, W. T., Akerlof, C. W., et al. 2004, AJ 127, 2436

Wils, P., Sodor, A., 2005, IBVS 5655, 1 